\title{
Hat Trick Observation for Bosons
}

\section{The CMS Collaboration confirms that certain particle-smashing events} produce three massive bosons.

\section{By Katherine Wright}

W

hen it comes to particle interactions, those involving massive bosons are some of the least well understood. A new observation from the CMS Collaboration at CERN could change that [1]. The team reports the production of three "gauge" bosons in proton-proton collisions at the Large Hadron Collider (LHC). These observations could provide a new window into how bosons interact and have been a long-term experimental quest of researchers. The results also appear in the collaboration's 1000th paper, giving additional cause for celebration.

The standard model includes four massive bosons: the gauge bosons $\left(W^{+}, W^{-}\right.$, and $\left.Z\right)$, which mediate the weak fundamental interaction, and the Higgs boson, which provides the gauge bosons with their mass. These bosons are all predicted to interact with each other, but for researchers to glean information about such interactions, they need to see several of these particles at once.

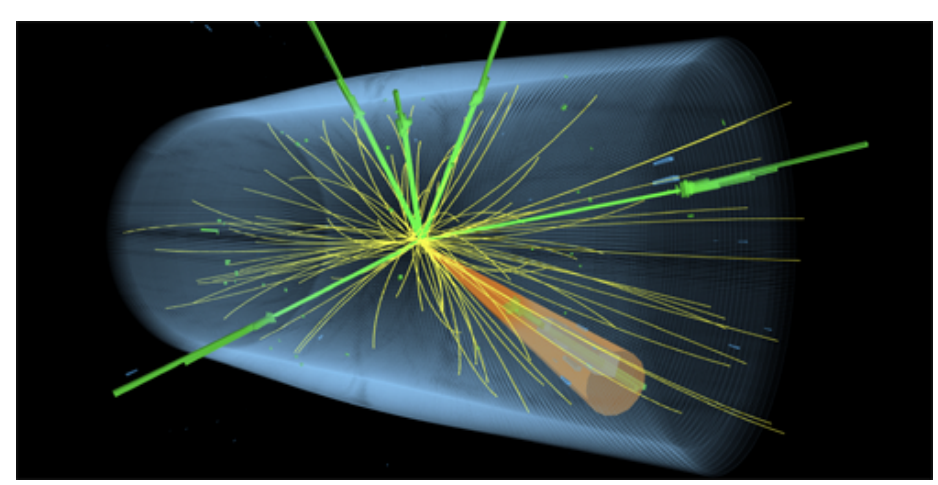

Credit: CMS Collaboration
One issue with witnessing these events is that only 1 in 100 trillion proton-proton collisions produces three gauge bosons simultaneously, meaning that researchers need to record a huge number of collisions. That happened in the 2016-2018 LHC data run, during which there were 15 quadrillion $\left(10^{15}\right)$ proton-proton collisions. Analyzing the data, the CMS Collaboration found signs that among the collisions were some that produced three gauge bosons-for example $W W W$ or WWZ-with a statistical significance of 5.7 standard deviations. Such events had been spotted before, a handful of times, but this high significance establishes the existence of these events beyond a doubt, the team says.

Katherine Wright is a Senior Editor for Physics.

\section{REFERENCES}

1. A. M. Sirunyan et al., "Observation of the production of three massive gauge bosons at $\sqrt{s}=13$ TeV," Phys. Rev. Lett. 125, 151802 (2020). 